\title{
Propuesta de acciones estratégicas para la implementación del modelo de negocio online en las agencias de la OSDE Viajes Cuba
}

Proposal of strategic actions for the implementation of the online business model in the agencies of the OSDE Viajes Cuba

Lic. Alfonso Lázaro Roche Góngora. ${ }^{1}$

\begin{abstract}
E-commerce is one of the fastest growing trends in the travel and tourism industry. In Cuba, the government travel agencies grouped in the OSDE Viajes Cuba have considered incorporating electronic commerce into their business model. However, there are still many limitations on the websites of these agencies and payment gateways have not been activated on most of these sites. Likewise, each agency separately carries out its own strategy for implementing the online business model.

This paper proposes a group of strategic actions to implement and perfect the online business model in OSDE Viajes Cuba travel agencies. For this, the best practices of the leading online travel agencies in the sector, the main trends related to electronic commerce in travel agencies and the experience accumulated by the OSDE Viajes Cuba agencies were taken into account.

Among the main proposals are: guidelines to follow for the design of websites and the selection of payment gateways, creation of an electronic commerce department within the agencies, development of online reputation management campaigns and social networks , establishment of a competitive pricing strategy, activation of loyalty programs, implementation of a collaboration strategy among Cuban travel agencies regarding electronic commerce, among others.
\end{abstract}

Key Words: Online Business Model, E-Commerce, OSDE Viajes Cuba, Online Travel Agency, OTA.

\section{Resumen}

${ }^{1}$ Profesor de la Facultad de Turismo de la Universidad de La Habana, alroche95@gmail.com 
El comercio electrónico es una de las tendencias con mayor auge en la industria de viajes y turismo. En Cuba, las agencias de viajes gubernamentales agrupadas en la OSDE Viajes Cuba se han planteado incorporar el comercio electrónico a su modelo de negocio. Sin embargo, todavía existen muchas limitaciones en los sitios web de estas agencias y no se han activado pasarelas de pagos en la mayoría de estos sitios. Asimismo, cada agencia por separado lleva a cabo su propia estrategia de implementación del modelo de negocios online.

El presente trabajo propone un grupo de acciones estratégicas para implementar y perfeccionar el modelo de negocios online en las agencias de viajes de la OSDE Viajes Cuba. Para ello se tuvo en cuenta las buenas prácticas de las agencias de viajes online líderes en el sector, las principales tendencias relativas al comercio electrónico en las agencias de viajes y la experiencia acumulada por las agencias de la OSDE Viajes Cuba.

Entre las principales propuestas se encuentran: pautas a seguir para el diseño de los sitios web y la selección de pasarelas de pagos, creación de un departamento de comercio electrónico dentro de las agencias, desarrollo de campañas de gestión de la reputación online y de redes sociales, establecimiento de una estrategia de precios competitiva, activación de programas de fidelización, implementación de una estrategia de colaboración entre las agencias de viajes cubanas con respecto al comercio electrónico, entre otras.

Palabras Clave: Modelo De Negocios Online, Comercio Electrónico, OSDE Viajes Cuba, Agencia De Viajes Online, OTA.

\section{Introducción}

A partir de la década de 1990, el mundo experimentó una verdadera revolución tecnológica con la aparición y rápida adopción de la internet como parte de la vida cotidiana. La gran mayoría de los negocios necesitaron adaptarse a este fenómeno en aras de sobrevivir, pues se ha mantenido in crescendo, de forma ininterrumpida, una tendencia del gran público a utilizar la internet, no solo para la búsqueda de información, sino cada vez más para concretar la compra de productos y servicios. Por tal motivo, el comercio electrónico ha cobrado un auge sin precedentes que ha llevado a muchas empresas a lanzarse al mundo digital para no perder un segmento de mercado cada vez mayor.

En el caso de Cuba, a pesar del desfase tecnológico asociado a la falta de infraestructura y recursos para invertir en las telecomunicaciones, muchas empresas se han sumado a la campaña internacional de conquista del ciberespacio. Además, el estado cubano, en especial desde el 2017, ha abogado por la incorporación del modelo de negocios online en los sectores que necesitan de él para su correcto posicionamiento y comercialización. Tal es el caso del sector del turismo, que ha logrado insertarse en el mundo digital. En este sentido, se ha desarrollado el portal de información al turismo y ventas online Cuba Travel, así como sitios web de hoteles, agencias de viajes, transportistas, restaurantes y cadenas hoteleras. 
Uno de los grupos empresariales más importantes para el turismo cubano es la OSDE Viajes Cuba, entidad que agrupa a las cuatro agencias de viajes principales del Ministerio de Turismo: Viajes Cubanacán, Cubatur, Ecotur y Havanatur. Si se tiene en cuenta el estado de los sitios web de las agencias de viajes citadas son evidentes un grupo de limitaciones de diseño, accesibilidad e inclusión del modelo de negocios online. La presente investigación plantea como su principal problema de investigación las siguientes cuestiones: las agencias de viajes del grupo Viajes Cuba se muestran rezagadas en comparación con sus competidores en los niveles internacional y regional debido al insuficiente grado de utilización del modelo de negocios online, que posee uno de los indicadores de crecimiento más elevados del sector. Esta situación provoca una ralentización en el crecimiento de las agencias vinculadas al grupo y constituye una amenaza en el medio y largo plazo para la supervivencia de las mismas si no se toman las medidas pertinentes.

Para dar respuesta a esta problemática el presente trabajo se traza como objetivo general:

- Proponer un grupo de acciones estratégicas que permita a las agencias de viajes del grupo Viajes Cuba la implementación del modelo de negocios online teniendo en cuenta las especificidades del contexto cubano actual.

- Los objetivos específicos de la investigación son:

- Investigar las principales tendencias en los niveles regional y mundial sobre el modelo de negocios online para las agencias de viajes.

- Establecer las acciones estratégicas de la propuesta en base a las buenas prácticas de las OTA líderes del sector, las principales tendencias relativas al negocio online para las agencias de viajes y la experiencia acumulada por las agencias de la OSDE Viajes Cuba respecto al comercio electrónico.

\section{Metodología}

La unidad de análisis del presente estudio es la OSDE Viajes Cuba y las diferentes agencias de viajes asociadas a la misma: Viajes Cubanacán, Cubatur, Ecotur y Havanatur. Las técnicas y métodos empleados se enfocaron en la obtención de información relativa a diferentes aspectos del comportamiento humano con el objetivo de implementar y perfeccionar el modelo de negocios online en las agencias de viajes del grupo Viajes Cuba.

Por otra parte, se llevó a cabo una búsqueda planificada y sistemática de información de carácter primario y secundario. Los datos obtenidos permitieron comprender el objeto de estudio y crear una propuesta de mejoras a partir del resumen del saber hacer de los líderes del sector de las agencias de viajes online y la experiencia acumulada por las agencias cubanas analizadas. La información primaria se alcanzó a partir de la observación del objeto de estudio y las entrevistas y consultas a expertos en la materia. Asimismo, la información secundaria se recuperó de bibliografía variada publicada por expertos en el tema de estudio, documentos rectores de las agencias de viajes estudiadas y análisis de páginas web. 
Los métodos y técnicas empleadas para la investigación fueron: histórico-lógico, análisissíntesis, inducción-deducción, enfoque a sistema, hipotético-deductivo, análisis bibliográfico, observación científica y entrevista a expertos

\section{Propuesta de acciones estratégicas para la implementación del comercio online en las agencias de viajes del grupo viajes Cuba.}

La presente propuesta de acciones estratégicas ha sido elaborada a partir de una búsqueda bibliográfica extensiva sobre el desarrollo del modelo de negocios online para las agencias de viajes. Asimismo, se han tenido en cuenta los resultados del trabajo de diploma "El modelo de negocios online para las agencias de la OSDE Viajes Cuba" (Roche, 2019) que abarca el análisis del saber hacer de las agencias de viajes online: Expedia, Booking, Despegar y Almundo y expone además las principales fortalezas y debilidades de las agencias de la OSDE Viajes Cuba con respecto al comercio electrónico.

A continuación, se analiza un grupo de líneas de acción para la implementación exitosa de un módulo de comercio online en las agencias de viajes del grupo Viajes Cuba.

\section{Diseño y gestión del sitio web}

En primer lugar, para la puesta en práctica de un módulo de comercio online, se debe garantizar el lanzamiento de un sitio web. La importancia de poseer un sitio web eficiente radica en que este constituye el punto neurálgico de la estrategia de comercio-e, pues la venta de los productos se concreta allí. Un sitio web de calidad debe cumplir los siguientes puntos:

1- Diseño atractivo y dinámico: el sitio debe ser visualmente atractivo para los visitantes en aras de garantizar su permanencia en él. De esta forma, se prioriza el contenido visual en forma de imágenes y videos que describan los productos y servicios que se ofrecen. Este contenido multimedia debe poseer un formato de alta calidad que resulte impactante para el cliente potencial e incite la compra de los productos. Por otra parte, los textos utilizados deben ser claros y sencillos, con un lenguaje correcto y accesible a todos los públicos.

2- Multilingüe: un sitio web publicado en varios idiomas es fundamental para atraer clientes de otras latitudes. En el caso de una agencia de viajes, el sitio debe estar publicado en los idiomas de los principales mercados emisores de la compañía.

3- Navegación intuitiva: el sitio debe poseer una usabilidad e interfaz sencillas para que los usuarios puedan consultar fluidamente los contenidos del sitio.

4- Capacidad multiplataforma: debe adaptarse al tamaño de pantalla de los diferentes dispositivos. De esta forma se garantiza la audiencia de los usuarios de teléfonos móviles, tabletas y otros dispositivos inteligentes.

5- Velocidad de carga: constituye un aspecto clave para evitar que los clientes salgan prematuramente del sitio sin haberlo visto siquiera. Los gestores del sitio y el equipo de soporte técnico deben seguir un grupo de buenas prácticas relativas a la optimización de contenidos para que las páginas carguen lo más rápido posible. 
6- Optimización SEO: este aspecto es imprescindible para el posicionamiento del sitio en los motores de búsquedas. De esta manera, se contribuye a que la agencia de viajes en cuestión aparezca en los primeros resultados de los buscadores principales como Google, Bing y Yahoo. De hecho, la consultora (Reason Why, 2018) asegura que el éxito de las agencias de viajes online se encuentra en aparecer en la primera página de Google.

7- Integración de un módulo B2B para facilitar el intercambio con los intermediarios del sistema de distribución turístico, en especial las agencias de viajes emisoras y los turoperadores. Según (Salgado, 2019) se debe considerar habilitar un acceso exclusivo al sitio para los clientes B2B o crear un sitio web especializado solamente en el comercio entre empresas.

8- Garantizar las características inherentes a la web 2.0 en el sitio para facilitar un intercambio de comunicación bidireccional entre la empresa, los clientes y los usuarios del sitio. Por ejemplo, se puede incluir la posibilidad de dejar comentarios sobre la experiencia del viaje en el propio sitio, crear una aplicación de atención directa en línea, habilitar un foro en el que los viajeros puedan intercambiar ideas entre ellos y con la empresa, entre otras funcionalidades.

\section{Activación de la pasarela de pago y otros medios de pago electrónico}

Para autores como (Quelle, 2017) y (Urbano, 2016) la activación de una pasarela de pago constituye otro de los requisitos básicos para la puesta en marcha de un módulo efectivo de comercio electrónico. Es gracias a los servicios de intermediación ofertados por estos proveedores que los clientes finales pueden contratar de manera fácil e intuitiva los diferentes productos online ofertados por la compañía. No obstante, en el caso de Cuba la selección y activación de una pasarela de pagos resulta complejo debido a las consecuencias del bloqueo estadounidense que impide a las principales pasarelas de pagos internacionales hacer negocios con Cuba.

Sin embargo, existen dos compañías que operan en el país que permiten las transacciones electrónicas desde bancos europeos hacia la Isla. Estas son Azuba y Bidaiondo, ambas radicadas en España. La primera de ellas cuenta con una experiencia más amplia en Cuba mas no posibilita las transacciones desde bancos estadounidenses; es decir, los ciudadanos norteamericanos no pueden utilizar los servicios de esta compañía para comprar productos turísticos cubanos de manera online. Por otra parte, Bidaiondo posee la ventaja competitiva de ofertar a las agencias de viajes que contraten sus servicios la posibilidad de comerciar con el mercado de Estados Unidos.

Por otra parte, las agencias de viajes estudiadas podrían negociar con los bancos cubanos la implementación de una pasarela de pago local que permita a los clientes nacionales comprar sus productos de manera online. Además, se podría aprovechar la iniciativa de la pasarela de pago Pasared, desarrollada por la Empresa de Tecnologías de la Información para la Defensa (Xetid) para que los clientes que cuenten con una tarjeta multibanca o telebanca puedan adquirir los diferentes productos ofertados por las agencias de viajes del grupo Viajes Cuba. 
Para ello, esta posibilidad de pago se habilitaría en los sitios de venta de las agencias, en las aplicaciones para móviles que se desarrollen y en otros sitios web del sistema turístico cubano que se creen para la venta a los clientes nacionales. Con esta tecnología, las agencias de la OSDE podrían satisfacer las necesidades del turismo nacional y desarrollar campañas de marketing para captar a este segmento de mercado. Además, esta iniciativa se traduciría en una reducción importante en sus costos de operación puesto que necesitarían menos personal para atender las ventas directas.

\section{Gestión de los recursos humanos}

El autor considera que la creación de un equipo de trabajo especializado en comercio online constituye una condición sine qua non para el éxito del módulo de comercio electrónico de las agencias de viajes de la OSDE Viajes Cuba. En el caso de una OTA, el capital humano contratado debe poseer un abanico de aptitudes y actitudes tales como conocimientos avanzados sobre comercio electrónico, marketing online, gestión de redes sociales, capacidad de trabajo en equipo y habilidades básicas de creación de sitios web y SEO. También debe contar con un marcado enfoque al cliente en aras de satisfacer y superar las expectativas de los consumidores. Por otra parte, los especialistas que trabajen directamente con los clientes deben poseer elevadas habilidades comunicativas y de redacción.

Con respecto al tamaño del área de comercio online, el autor considera que debe ser directamente proporcional al éxito del modelo, por lo que puede variar desde una unidad casi virtual hasta una UEB independiente. En la medida en que crezca y se consolide el nuevo módulo de e-commerce se deben contratar a un mayor número de especialistas que gestionen funciones más específicas y sean capaces de brindar una atención cada vez más rápida y eficiente a las solicitudes de los clientes.

Además, el autor recomienda que las agencias de la OSDE habiliten un puesto de trabajo para un máximo responsable de comercio-e. En dependencia del tamaño que adquiera el departamento de comercio online y la carga de trabajo del jefe del equipo el puesto podría evolucionar hasta un subdirector o director de comercio-e. Este directivo deberá contar con los niveles de autorización suficientes para desarrollar sin trabas el comercio electrónico en la empresa.

Por otra parte, el autor considera que la función de creación de paquetes y productos turísticos especializados para los clientes online debe ser llevada a cabo dentro del departamento de comercio-e. De esta manera se garantizaría una respuesta rápida a las solicitudes de los clientes, aspecto que constituiría una ventaja competitiva para la empresa. Lógicamente, para el desarrollo eficiente de esta función los especialistas del departamento deben tener acceso a las capacidades contratadas de la agencia en tiempo real.

También, desde el área de comercio electrónico se deberá llevar a cabo la función de gestión de redes sociales. Para ello será necesario contratar a un especialista con 
formación como community manager. Esta persona será la encargada de convertir a las redes sociales en herramientas de promoción, comunicación, retroalimentación y canales de venta.

\section{Herramientas y estrategias propuestas para la implementación del comercio-e}

\section{Gestión de la reputación online y estrategia de marca}

Una de las acciones indispensables para el desarrollo de una campaña de gestión de la reputación online es activar en los sitios web de las agencias de viajes cubanas un sistema de opiniones que permita a los usuarios emitir sus criterios sobre los servicios y paquetes contratados. También resulta recomendable habilitar la posibilidad de compartir contenido multimedia para los usuarios del sitio. De esta manera se promueve la generación de contenidos en forma de imágenes y vídeos en el sitio web institucional.

Por otra parte, es necesario prestar especial atención a la reputación online en los sitios de opiniones de viajeros, como Tripadvisor. Esta práctica resulta particularmente importante porque las opiniones de estos sitios no pueden ser intermediadas por la compañía. Asimismo, se deberá incitar a los clientes a compartir sus experiencias en estas redes y responder a la mayor cantidad posible de opiniones de viajeros, en especial aquellas opiniones que meriten una disculpa o un agradecimiento especial (Craig \& Edward, 2014).

Otra de las pautas esenciales para establecer la campaña de gestión de la reputación online es una estrategia preconcebida ante situaciones de crisis. Resulta prácticamente imposible satisfacer a todos los clientes de la compañía y siempre habrá algunos que no estarán felices con su experiencia y publicarán opiniones negativas en las redes sociales. Los especialistas del departamento de comercio-e deben crear con anterioridad un plan de acción ante casos de crisis de comentarios negativos. Como regla general, las respuestas oficiales deben ser empáticas, respetuosas y coherentes con los aspectos criticados. Para (Velázquez, 2019), las reglas básicas para la gestión de comentarios negativos son: evitar tiempos largos de respuesta, no ignorar el problema y no limitar la comunicación. También (López, 2019) asegura que se le debe dar respuesta al 100\% de los comentarios negativos en un periodo de tiempo corto. Con esta práctica se evitan repeticiones de evaluaciones negativas y la empresa gana en credibilidad online. Además, han de analizarse las causas de las experiencias negativas y corregir los errores detectados.

Asimismo, para la administración del brazo online de las agencias de viajes cubanas se debe velar por la promoción y consolidación de la marca que representa a cada agencia en el sector de los viajes. En este sentido, debe existir una cohesión entre los departamentos online y offline para defender a la marca, ya que ambos utilizarán el mismo nombre comercial. Para el departamento online, resulta particularmente importante que la marca adquiera una reputación de confiabilidad, seguridad, profesionalidad, calidad en los servicios, adecuada relación calidad-precio y eficiente sistema de atención al cliente. 
De esta manera, a medida que el brazo online de la agencia se desarrolle, la marca adquiriría mayor prestigio y las ventas globales aumentarían.

\section{Gestión de redes sociales}

Para la gestión de las redes se recomienda la creación de perfiles institucionales en las principales redes sociales de la actualidad como Facebook, Instagram, YouTube, LinkedIn, Twitter y Pinterest. Los especialistas de cada agencia decidirían qué perfiles institucionales abrir en dependencia de su popularidad en los principales mercados de la empresa.

Es importante destacar que cada red social se enfoca en tipos de contenido diferentes, por lo que las publicaciones deben ser coherentes con el formato de la red social. Por ejemplo, Facebook se utiliza con frecuencia para publicar contenido diverso que combine fotografías con textos y videos. Por otra parte, Instagram y Pinterest son redes más visuales por lo que la calidad de las fotografías empleadas debe ser elevada. Asimismo, LinkedIn pudiera ser utilizada para publicar y compartir contenidos relevantes del sector de las agencias de viajes. Con esta práctica se afianza la posición de la empresa como especialista en el área. En el caso de Twitter, si bien ha perdido mucha popularidad se utiliza todavía para compartir información en tiempo real y lanzar encuestas.

Para garantizar la efectividad de las redes sociales debe existir a nivel de departamento un conjunto de objetivos que permitan medir cuantitativa y cualitativamente el alcance de estas herramientas. Asimismo, los contenidos a publicar deben ser planificados con antelación de forma que se garantice una periodicidad marcada en las publicaciones. Por tal motivo, es fundamental la creación de un calendario editorial para subir contenidos a la web. Cabe destacar que toda la información compartida no debe ser sobre los productos y servicios de la agencia. Se debe promover también información vinculada al sector de los viajes que resulte relevante para los públicos objetivos de la empresa. De hecho, especialistas en el sector del marketing digital y la gestión de redes sociales, como (Calcaneo, 2018), (Santos, 2018), (Quero, 2018) y (Guibert, 2018) recomiendan que la mayor parte del contenido posea información valiosa para el cliente y la menor parte describa y promocione la marca o los productos de la empresa.

Por otra parte, el autor recomienda al personal encargado de la gestión de redes sociales que considere la factibilidad de adquirir herramientas de pago para la gestión de redes sociales. De esta manera se facilitaría la labor del community manager, puesto que tendría acceso a la automatización de los contenidos, al monitoreo de las redes sociales y a los informes generados por estas herramientas. Algunas de las plataformas más populares para la gestión de redes son: Hootsuite, Buffer y Metricool (Websa100, 2018).

En el nivel de la OSDE se recomienda la creación de un protocolo de evaluación y control de la gestión de las redes sociales de las diferentes empresas asociadas al grupo. Para ello, se deben crear indicadores de control y contratar personal especializado en la administración y gestión de redes. Por ejemplo, un social medio manager sería el individuo encargado de elaborar las directivas de gestión y calidad que los community managers de cada agencia deberían seguir. Esta persona podría monitorear también la 
evolución de los perfiles institucionales de cada agencia, trazar estrategias generales para su mejora y proponer cambios pertinentes.

\section{Estrategia de precios competitiva}

Para gestionar la variable precio, el autor de este trabajo recomienda que el departamento de comercio online establezca valores inferiores, o al menos similares, a los precios finales de los turoperadores. De esta manera, se promueven las ventas directas y se reduce la dependencia de los canales tradicionales. Asimismo, se garantiza que los sitios de las agencias de viajes cubanas adquieran la reputación de poseer buenas ofertas que a la larga afianzarán el comercio-e como modelo de negocios. Además, durante la temporada baja se pueden lanzar ofertas especiales directas al cliente final que sean rentables tanto para los prestatarios locales como para las agencias cubanas.

Una política de precios inferiores a los ofertados a los intermediarios dependerá de los contratos específicos que se hayan concertado con estos. En algunas ocasiones los intermediarios presentan cláusulas en los contratos que obligan a las agencias de viajes a brindar un precio inferior o similar al precio ofertado al resto de los canales de distribución. De esta manera, ante posibles violaciones, las agencias cubanas podrían sufrir reclamaciones o los contratos con diversos proveedores podrían ser anulados. Ante esta coyuntura se deben analizar cuidadosamente los contratos ya existentes con los diversos turoperadores y evaluar sus posibles reacciones ante esta nueva estrategia.

En el corto y mediano plazo, los intermediarios deberían jugar un papel preponderante, pero en el largo plazo, las ventas directas representarían un incremento significativo en las ganancias de las agencias de viajes cubanas. De esta manera, se debe planificar en el largo plazo una mayor independencia de los proveedores tradicionales a partir de un incremento significativo del módulo de comercio-e. Por otra parte, si se tiene en cuenta a Cuba como destino-país y al Ministerio de Turismo como organismo rector de la OSDE Viajes Cuba, se evidencia que ante una mayor independencia de los canales tradicionales disminuye el riesgo asociado a las transacciones con terceros. Además, se contribuiría a mejorar los macro indicadores turísticos como ingresos totales al país, ingresos por turista y gasto medio por turista.

\section{Programa de fidelización para clientes repitentes}

Los clientes fidelizados favorecen la disminución de costos por concepto de marketing y publicidad. Asimismo, constituyen un segmento con el que se puede predecir de manera más sencilla las ventas de las empresas. Además, un cliente repitente se convierte en la mayoría de los casos en un agente de ventas para la empresa a partir de la comunicación boca-oreja. De esta manera, el cliente satisfecho compartirá su opinión en las redes sociales y aconsejará a sus familiares y amigos la compra de los mismos productos que fueron de su agrado (Velázquez, 2019).

Como se abordó en el epígrafe anterior, con las ventas directas a través de los sitios web de las agencias cubanas se puede brindar precios más económicos a los clientes finales. 
Asimismo, se pueden ofrecer rebajas a los clientes que hayan reservado con anterioridad en los sitios web de las agencias. Esta reducción en el precio no implicaría una disminución en los ingresos de la agencia, puesto que la reducción del precio sería proporcional a las comisiones que adquieren los turoperadores por la venta de los productos cubanos. Esta medida daría paso a un programa de fidelización para clientes repitentes.

Uno de los métodos más empleados para promover la lealtad de los clientes hacia las empresas es la utilización de códigos promocionales. Con esta técnica se puede otorgar a los clientes acceso a enlaces especiales hacia el sitio web que le ofrezcan un determinado porcentaje de descuento. Este beneficio sería válido por un determinado marco de tiempo y podría ser utilizado por familiares o amigos de los antiguos clientes de la empresa. También es posible complementar la estrategia de fidelización con la de gestión de la reputación online. Por ejemplo, se les puede otorgar descuentos especiales a clientes que compartan su experiencia de viajes en sitios de opiniones.

Por otra parte, se puede promover la fidelización de los clientes si se les otorgan beneficios extras a aquellos que adquieran con frecuencia los productos de la agencia. Estos beneficios estarían en función de la estrategia particular de cada empresa. Por ejemplo, se les podría brindar acceso a información exclusiva; a un número telefónico de asistencia especializada disponible 24 horas; a descuentos especiales ante fechas significativas como cumpleaños, aniversarios de bodas, lunas de miel y viajes en grupo; entre otros beneficios.

Cabe destacar que un programa de fidelización permitiría hacer frente a la competencia que utiliza medidas de este tipo para retener a los clientes. De esta manera, las agencias de viajes cubanas adquirirían una ventaja competitiva. Además, esta estrategia resultaría muy positiva no sólo para las agencias de viajes sino para toda la industria turística cubana en general que se beneficiaría indirectamente con la venta de sus capacidades por parte de las agencias.

\section{Estrategia de colaboración entre las agencias de viajes del grupo viajes Cuba}

El autor de este trabajo recomienda a la OSDE Viajes Cuba la implementación de una estrategia de colaboración entre las diferentes agencias de viajes para el desarrollo del modelo de negocios online. En reiteradas ocasiones se ha puesto en evidencia por parte de las diferentes agencias vinculadas a la OSDE la existencia de un espíritu competitivo. Sin embargo, el autor considera que esta mentalidad pudiera ser perjudicial para las agencias, puesto que la competencia más significativa de la OSDE se encuentra fuera de la misma y no entre sus propias agencias. De esta forma se evitan gastos materiales y humanos dentro de las agencias del grupo que busquen resaltar sobre el resto de los integrantes de la OSDE. Este esfuerzo pudiera ser canalizado hacia estrategias conjuntas que permitan elevar el sector de las agencias de viajes cubanas a una posición destacada en el mercado latinoamericano.

Para fomentar la cooperación entre las agencias en el campo del comercio-e se pueden establecer talleres de intercambio con una periodicidad trimestral o semestral para 
compartir las experiencias y buenas prácticas de cada compañía en el sector. De esta manera se lograrían estrategias comunes para desarrollar el modelo de negocios online. Asimismo, las agencias podrían incorporar en cada uno de sus sitios web los productos ofertados por los otros miembros del grupo. De esta forma se promueven las ventas totales de la OSDE y se enriquece la cartera de productos online de todos los sitios. Lógicamente, cada agencia por separado decidiría qué producto de las otras empresas incorporar al sitio en dependencia de los mercados objetivos de cada una.

Por otra parte, las agencias de la OSDE Viajes Cuba podrían expandir el alcance de la colaboración hacia el resto de las agencias de viajes gubernamentales cubanas. De esta forma, se establecerían vínculos de cooperación con Paradiso, Gaviotatur y San Cristóbal. Con esta iniciativa, se podrían recuperar experiencias mutuamente beneficiosas para cada compañía.

\section{Utilización eficiente del gds amadeus}

Un empleo adecuado de las tecnologías y la infraestructura del GDS Amadeus puede convertirse en una ventaja competitiva para las agencias de la OSDE Viajes Cuba, puesto que este GDS es el líder en tecnología y distribución turística en el nivel mundial. En la actualidad, todas las agencias de la OSDE han establecido convenios con el grupo, por lo que tienen acceso a las capacidades de alojamiento, transporte terrestre y boletería aérea listadas en el sistema a través de la aplicación Amadeus Selling Platform. Con esta tecnología, las agencias cubanas pueden chequear en tiempo real los precios y disponibilidad de algunas de las principales cadenas hoteleras y de la mayoría de las aerolíneas que operan en Cuba, así como realizar la reserva y/o venta de esos productos.

En el departamento de comercio online, la tecnología Amadeus sería especialmente útil para los clientes que soliciten modalidades de viajes multidestinos. De esta manera, los especialistas de comercio-e podrían crear paquetes turísticos que combinen las capacidades de Cuba con las ofertas de otros destinos turísticos internacionales. Estas opciones se podrían presentar como productos establecidos en el sitio web o como una posibilidad de viaje a la carta. La implementación de esta recomendación dependería en última instancia del objeto social de las diferentes agencias del grupo y su estrategia corporativa.

Por otra parte, se recomienda a las agencias de la OSDE Viajes Cuba que integren la tecnología de Amadeus en sus sitios web. De esta manera podrían ofertar la venta de boletería aérea nacional e internacional desde el propio sitio de ventas. Con esta práctica crearían productos turísticos de mayor complejidad y atractivos para los clientes online combinando la venta de boletería aérea con alojamiento, excursiones y transporte en el destino. Esta sinergia entre Amadeus y los sitios web de las agencias de Viajes Cuba aportaría un importante valor añadido a los clientes que se traduciría en un incremento significativo de las ventas online. Además, esta práctica se podría hacer extensiva a las representaciones de las agencias cubanas de la OSDE en el exterior. 
Para facilitar la incorporación de esta tecnología, se recomienda que las agencias de la OSDE contraten la herramienta Amadeus e-Power. Esta solución integra en los sitios web los productos de Amadeus Selling Platform con los productos propios de la agencia o aquellos ofertados por terceros. Además, la empresa puede elegir los productos a mostrar en dependencia de sus intereses comerciales. Además, las agencias de viajes cubanas podrían distribuir a través de Amadeus las capacidades de la empresa de ómnibus locales Viazul y de esta manera comercializar desde sus páginas de venta la transportación hacia los diferentes destinos turísticos cubanos.

\section{Estrategia de comunicación multicanal}

Para conectar y comunicarse con los clientes de la empresa, el autor de este trabajo recomienda que se habiliten varios canales de comunicación. De manera tradicional, se ha utilizado el e-mail para el intercambio de información entre los clientes y las agencias de viajes. Más recientemente, con el aumento de la penetración de Internet y el auge de las redes sociales, se ha creado un grupo de métodos de comunicación que han adquirido gran relevancia. Por ejemplo, la mensajería instantánea a través de plataformas como Messenger y WhatsApp es muy utilizada por los usuarios digitales de la actualidad. También, las llamadas telefónicas o videollamadas a través de las plataformas de Internet resultan una forma de contacto accesible debido a su bajo costo y amplia usabilidad. Por tal motivo, Skype y las funciones de llamadas de WhatsApp han ganado un rol preponderante en un gran número de países.

Por otra parte, la creación de grupos en redes sociales o en plataformas de comunicación podría constituir un método para hacer llegar a los mismos mensajes a un grupo de personas afines y crear una comunidad online fidelizada a las ofertas de la agencia. En este sentido se deben explotar las potencialidades que ofrecen plataformas como Facebook, Instagram, WhatsApp, entre otras.

Además, el autor recomienda crear canales de comunicación y retroalimentación a partir de boletines electrónicos. Esta opción se puede habilitar desde el sitio web institucional y también se pueden emplear los correos electrónicos recuperados por el departamento de comercio-e para comunicarse con los clientes. También resulta necesario solicitar la autorización de los clientes antes de emitir mensajes por esta vía. Con esta práctica se evita el uso abusivo de la información brindada por los clientes y se combate el spam.

\section{Implementación de una política de mejora continua}

Una política de mejora continua permitiría al departamento de comercio-e de las diferentes agencias del grupo perfeccionar la gestión del brazo online de la compañía y adquirir renombre internacional. Esto se lograría al implementar estándares de calidad cada vez más elevados que garanticen superar las expectativas de los clientes.

La realización de encuestas constituye una de las prácticas adecuadas para medir el nivel de satisfacción de los clientes y retroalimentarse con sus opiniones. Para ello se pueden enviar formularios de correo electrónico a los clientes de las empresas o activar encuestas en los sitios web. 
Por otra parte, el estudio constante de las variables del entorno facilitaría a las agencias del grupo la detección de los cambios y tendencias fundamentales en el sector y adaptarse consecuentemente a ellos. Esta práctica resulta particularmente importante en el caso de las agencias de viajes online, pues la tecnología de los viajes evoluciona rápidamente y el cliente online se vuelve cada vez más exigente. Asimismo, esta técnica facilitaría la captación de nuevos mercados o clientes más rentables.

También se deben tener en cuenta las estrategias y mejores prácticas de los líderes del sector. Por tal motivo, se recomienda realizar estudios constantes de benchmarking para perfeccionar la capacidad operativa de la empresa. De esta manera, se garantiza el emparejamiento de los estándares de las agencias de la OSDE con los de las agencias de viajes online con más prestigio en los niveles regional y mundial.

\section{Creación de aplicaciones para teléfonos móviles}

Otra de las prácticas recomendadas para las diferentes agencias de viajes del grupo es la creación de aplicaciones especializadas para teléfonos móviles. Esta tecnología facilitaría a los clientes la navegación y compra de productos y servicios a través de una interfaz amigable. Es necesario destacar que el móvil constituye en la actualidad una de los medios más utilizados por los usuarios digitales para la consulta de información y reserva de productos turísticos. Además, una vez instalada la aplicación, la agencia adquiriría una visibilidad mucho mayor puesto que los clientes observarían la marca de la empresa más a menudo en su propio teléfono.

Para la activación de una aplicación para móviles se recomienda contratar los servicios de una empresa tecnológica que garantice altos estándares de calidad. Además, es necesario la actualización periódica del software para incluir nuevas prestaciones o garantizar la compatibilidad con los nuevos sistemas operativos. Asimismo, es importante que la aplicación sea compatible tanto con el sistema Android como IOS.

A partir de las aplicaciones móviles especializadas se pueden poner en marcha programas de fidelización que brinden promociones o regalos a los clientes de las empresas. También, se podría activar un módulo de localización en la aplicación que funcione con la dirección IP de los clientes o el GPS del teléfono. De esta forma, se podría habilitar la generación de ofertas automáticas a los clientes que se encuentren en un sitio determinado. Por ejemplo, si un cliente llega a uno de los destinos turísticos cubanos se le podría recomendar un grupo de excursiones locales.

\section{Integración del módulo de online con los sistemas de gestión de las AAVV}

La integración del módulo de ventas online con el sistema de gestión de agencias de viajes es una funcionalidad importante para el modelo de negocios online de las agencias de la OSDE Viajes Cuba. Esta práctica permitiría procesar de manera automática las reservaciones online en el sistema de gestión comercial y contable de la empresa. De otra manera, esta información deberá ser tabulada de forma manual con todos los costos asociados a los recursos humanos y posibles errores. Asimismo, una integración más 
amplia posibilitaría incluso actualizar de forma automática los precios de la página ante cambios en los precios de los proveedores.

Para llevar a cabo esta tecnología es necesario vincular el procesamiento de datos ofertado por las pasarelas de pago activas en el sitio con el sistema de gestión de agencias de viajes utilizado en la OSDE: el e-travel. Además, la integración debe ser compatible con el sistema contable que utilice cada empresa. En este sentido, se deberá contratar con los proveedores tecnológicos de cada agencia una solución que facilite este cambio.

\section{Creación de un plan de marketing digital}

En los acápites anteriores se han analizado diversas herramientas para potenciar el desarrollo del módulo de comercio online en las agencias de viajes del grupo Viajes Cuba. Desde un enfoque de marketing, muchas de ellas pueden ser utilizadas como medios de marketing digital, en especial: la gestión de redes sociales y la reputación online, la estrategia de precios competitiva, los programas de fidelización de clientes, la comunicación multicanal, los elevados estándares de atención y enfoque al cliente, el empleo de encuestas en el sitio para la mejora de productos y servicios, el estudio de las variables de cambio del entorno, el análisis de las prácticas de la competencia, el diseño óptimo de los contenidos para incitar a la venta, entre otros.

En este sentido, el autor de este trabajo recomienda a las agencias del grupo la creación de un plan de marketing digital adaptado a cada empresa que incluya las herramientas de la presente investigación que mejor se adapten a su estrategia corporativa. De esta manera, las agencias serán capaces de potenciar el empleo eficiente de estos métodos y definir sus principales objetivos a alcanzar en el área de la comercialización online. Por tal motivo, el plan de marketing digital funcionaría como un medio para medir el desarrollo del módulo de comercio online y aplicar medidas correctivas cuando resulte necesario o no se cumplan los objetivos previstos.

\section{Conclusiones}

Con la realización de la presente investigación el autor de este trabajo ha arribado a las siguientes conclusiones:

- Las acciones estratégicas propuestas relativas al diseño y gestión del sitio y la organización de los recursos humanos en un nuevo departamento de comercio online que integre la función productora y asesora permitirán impulsar considerablemente el desarrollo del comercio-e en las agencias de la OSDE Viajes Cuba.

- Las técnicas de gestión presentadas, tales como el monitoreo de la reputación online y las redes sociales, la implementación de precios competitivos, la colaboración entre las agencias del grupo, la creación de programas de fidelización, entre otras, facilitarán a las agencias de la OSDE incrementar sus ventas y alcanzar los estándares de calidad de las OTA líderes del sector. 
- Amadeus es el grupo líder en tecnología de viajes y turismo en el mundo y las agencias de la OSDE Viajes Cuba tienen contrato con él. La integración de las ofertas de vuelos, asientos de ómnibus y otros productos del GDS Amadeus en los sitios de venta electrónica es posible y ampliaría las ventas en esos sitios, las agencias y sus representaciones en el exterior.

\section{Referencias bibliográficas}

Calcaneo, I. (23 de Noviembre de 2018). Mailclick. Obtenido de El community mánager idóneo: quién es y cómo elegirlo. (Guía del 2019): htpps://www.mailclick.com.mx/community-manager

Craig, \& Edward, D. (7 de Enero de 2014). Reviewpro. Recuperado el 19 de Marzo de 2019, de https://www.reviewpro.com/es/blog/como-gestionar-y-responderopiniones-en-otas/

Guibert, Y. (2018). Hubspot. Recuperado el 23 de Abril de 2019, de htpps://bolg.hubspot.es/marketing/razones-para-tener-un-community-manager

López, C. (9 de Mayo de 2019). Optimización de la experiencia del Huésped. Casos de Éxito. La Habana, Cuba: Conferencia de la expo de tecnología de FITCUBA 2019.

Quelle, L. (28 de Abril de 2017). ¿Por qué elegir una pasarela de pagos? Recuperado el 13 de Marzo de 2019, de Ecommerce News: www.ecommerce-news.es/elegiruna-pasarela-de-pagos-59159

Quero, J. (Enero de 2018). jessicaquero.com. Recuperado el 23 de Abril de 2019, de Guía del community manager para principiantes: htpps://jessicaquero.com/guiacommunity-manager-principiantes/

QUIWIQ Marketing Online. (2019). QUIWIQ Marketing Online. Recuperado el 23 de Abril de 2019, de Servicio de gestión de redes sociales: https://quiwiq.com/servicio-gestion-redes-sociales

Reason Why. (24 de Octubre de 2018). Reason Why. Recuperado el 18 de Marzo de 2019, de El éxito de tu negocio está en la primera página de Google. El caso de booking.com.: https://www.reasonwhy.es/actualidad/el-exito-de-tu-negocio-estaen-la-primera-pagina-de-google-el-caso-de-bookingcom-2018-10

Roche, A. (2019). El Modelo de Negocios Online para las agencias de viajes de la OSDE Viajes Cuba. La Habana: Tesis en opción al grado de licenciado en turismo.

Salgado, J. E. (2008). El negocio electrónico en el sector del turismo. La Habana. 
Salgado, J. E. (18 de Mayo de 2019). Consideraciones sobre el comercio electrónico en Cuba. (A. Roche, Entrevistador) Miramar Trade Center, Edif. Santiago, 2do Piso, Oficina 2014, Ave 5ta e/ 76 y 78, Playa, La Habana, Cuba.

Santos, B. (29 de Enero de 2018). Hotmart. Recuperado el 23 de Abril de 2019, de ¿Cómo hacer una buena gestión de redes sociales?: https://blog.hotmart.com/es/gestionde-redes-sociales/

Urbano, S. M. (31 de julio de 2016). ¿Qué es y cómo funciona la pasarela de pago en ecommerce? Recuperado el 13 de Marzo de 2019, de Actualidad Ecommerce: www.actualidadecommerce.com/que-es-y-como-funciona-la-pasarela-de-pagoen-ecommerce/

Vázquez-Casielles, R., Belén, A., \& Suárez-Álvarez, L. (2009). Las agencias de viajes virtuales: ¿Cómo analizar la calidad de e-servicio y sus efectos sobre la satisfacción del cliente? Universia Business Review, 122-143.

Velázquez, A. (9 de Mayo de 2019). El comercial 2.0. Herramientas para la fidelización de los clientes digitales. . La Habana, Cuba: Conferencia de la expo de tecnología de FITCUBA 2019.

Websa100. (10 de Diciembre de 2018). Websa100. Recuperado el 24 de Abril de 2019, de Herramientas para Community Manager: https://www.websa100.com/blog/herramientas-para-community-manager/

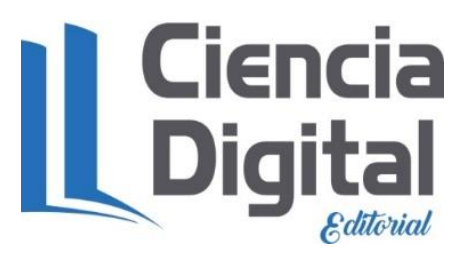




\section{PARA CITAR EL ARTÍCULO INDEXADO.}

Roche Góngora, A. L. (2021). Propuesta de acciones estratégicas para la implementación del modelo de negocio online en las agencias de la OSDE Viajes Cuba. Explorador Digital, 5(1), 201-217. https://doi.org/10.33262/exploradordigital.v5i1.1498

\section{¿Ciencia}

El artículo que se publica es de exclusiva responsabilidad de los autores y no necesariamente reflejan el pensamiento de la Revista Explorador Digital.

El artículo queda en propiedad de la revista y, por tanto, su publicación parcial y/o total en otro medio tiene que ser autorizado por el director de la Revista Explorador Digital.
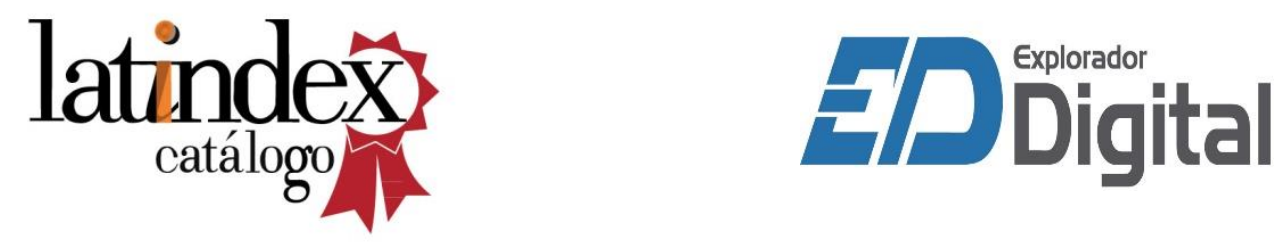